\title{
CIRED 2019: Bericht über Session 3 - Betrieb, Steuerung und Schutz
}

\author{
A. Abart OVE \\ Online publiziert am 10. Oktober 2019 \\ (C) Springer-Verlag GmbH Austria, ein Teil von Springer Nature 2019
}

Im Herbst 2018 wurden rund 450 Abstracts für Session 3 CIRED 2019 eingereicht. Insgesamt ist dabei zu beobachten, dass der Anteil der Arbeiten in guter Qualität stark zugenommen hat. Die hohe Anzahl an Einreichungen ebenso wie auch die Vielfalt der Themen zeigen die aktuelle Innovation aber auch den gleichzeitig einen enormen Entwicklungsbedarf in den Bereichen Betrieb, Steuerung und Schutz für Verteilernetze. Um die Konferenzbeiträge überschaubar zu halten, wurde vom Team Session 3 unter Vorsitz von Prof. Markus Zdrallek (Universität Wuppertal) in Abstimmung mit den Nationalkomitees rund ein Drittel der vorgeschlagenen Arbeiten für einen Konferenzbeitrag ausgewählt. Dabei mussten leider auch interessante Einreichungen abgelehnt werden. Von 180 ausgewählten Abstracts wurde im Jänner schließlich 156 „full paper" eingereicht - acht davon wurden von österreichischen Autoren. 24 der Beiträge wurden in den vier Blöcken der Main Session präsentiert und mehrere wissenschaftlich tiefer gehende Arbeiten im „Research and innovation Forum". Die meisten Autoren nutzten die Gelegenheit Ihre Arbeit bei einer der acht Poster-Touren von Session 3 zu präsentieren.

Dem Thema Betrieb wurden in der Main Session zwei Blöcke gewidmet. Der Einsatz von Augmented Reallity z. B. bei Schalthandlungen und Prüfungen von Sekundärtechnik wie auch der Einsatz künstlicher Intelligenz in der Zustandsbeurteilung sowie MonitoringFunktionen von Smart Metringsystemen haben Einblick in den künftigen Alltag der in der Fläche tätigen Mitarbeiter von Verteilernetzbetreibern geboten. Diesen Themenfeldern war auch der Round Table 6 "Grid Operation 2030 - Digitalisation of Distribution System Operation" gewidmet, bei dem die Teilnehmer am Podium in den neuen Technologien grundsätzlich ein hohes Potenzial sahen. Die Hürden im Bereich Datenschutz und Komfort, so war man sich am Podium einig, sind überwindbar, erfordern aber die Einbindung der betroffenen Netzbenutzer

Zum Großstörungsmanagement eingebrachte Beiträge beinhalten verbesserte Wetterprognosen aber auch sogar Vorhersage von Ausfällen, auf Grund derer Personal und Materialkapazitäten rechtzeitig optimal angepasst werden können.

Zum Themenbereich Schnittstellen und Koordination zwischen Übertragungs- und Verteilernetzbetreibern wurden Beiträge betreffend Großstörungsmanagement sowie Flexibilität und Blindleistung im Zusammenhang mit Engpassmanagement geboten. Letzteres wurde auch im Zusammenhang mit der Spannungshaltung in Verteilernetzen betrachtet. Im Rahmen des Round Table 4 „DSO/TSO Interactions" waren sich alle Teilnehmer am Podium einig, dass die Bedeutung der Verteilernetze zunimmt, da die Erzeugung und die Flexibilität zunehmend in den Verteilernetzen zu finden ist. Früher oder später ist für Verteilernetzbetreiber mit mehr Verantwortung für das Gesamtsystem zu rechnen.

Besonders beachtenswert ist auch ein provokanter Beitrag zweier Niederländer, in dem Überspezifikation begründet durch fehlendes
Knowhow bei Netzbetreibern, Behörden und Industriepartnern an Beispielen dargestellt wurde.

Im Bereich Netz-Steuerung handelten die meisten Beiträge von Automatisierung der Mittelspannungs- und Niederspannungsnetze bzw. selbstheilenden Netzen. Für Mittelspannungsnetze behandelten mehrere Beiträge sogenannte "Soft open points (SOP)" als Ersatz für herkömmliche Schalter, die es erlauben Lastflüsse zu beeinflussen. Dadurch werden der Betrieb in geschlossenen Ringen, eine höhere Versorgungsqualität und optimierte Nutzung des Netzes möglich. Gegenüber CIRED 2017 zeigte sich das Thema SCADASysteme etwas rückläufig ist. Sehr stark vertreten war dagegen das Thema Inselbetrieb und dazu verbreitet aufgebauten Pilotanwendungen. Eine breite Anwendung scheint aber noch eher in Ferne. In diesen Themenbereich fällt auch die als „Best Young Academic Paper Award Winner of Session 3" ausgewählte Einreichung, erstellt von Sebastian Palm (DE).

Im Block Schutztechnik war der Erdschluss, dessen numerische Modellierung und Ortung eines der beherrschenden Themen. Beim Round Table 2 kamen die führenden Experten zur Sternpunktsund Fehlerbehandlung zusammen und ließen die jahrzehntelange Entwicklung auf diesem Gebiet Revue passieren. Es bestand Einigkeit, dass trotz erheblicher Fortschritte noch weiterer Forschungsbedarf besteht. Insbesondere gibt es bei der Ortung vielversprechende Innovationen, die künftig eine Schutzabschaltung der betroffenen Hochspannungsleitung oder des Mittelspannungsleitungsabschnitts ermöglichen könnten. Aber auch im Bereich Kurzschlüsse wird über verbesserte Fehlererkennung und -ortung vor allem für Mittel- und Niederspannungsnetze berichtet. Die Weiterentwicklungen der Kommunikationstechnik, insbesondere 5G, ergeben zunehmend eine Integration der Schutztechnik in SCADA-Anwendungen der Netzautomatisierung. Dadurch können zunehmend selbstheilende Netze aufgebaut werden.

CIRED 2019 hat die hohe Innovationsbereitschaft der Verteilernetzbetreiber insbesondere auf den Gebieten Ancillery Services, Inselbetrieb, Erdschlussortung, Automatisierung in Mittel- und Niederspannungsnetzen gezeigt, sodass wir mit Spannung die im Jahr 2021 in Genf stattfindende CIRED erwarten können.

Hinweis des Verlags Der Verlag bleibt in Hinblick auf geografische Zuordnungen und Gebietsbezeichnungen in veröffentlichten Karten und Institutsadressen neutral.

$25^{\text {th }}$ International Conference on Electricity Distribution, Madrid, Spanien, 03. bis 06. Juni 2019.

http://www.cired2019.org/images/Executive_summary_CIRED2019_Session3.pdf.

Abart, Andreas, Netz Oberösterreich GmbH, Neubauzeile 99, 4030 Linz, Österreich (E-Mail: andreas.abart@netzooe.at) 